\title{
A new generalization of Radon's Inequality and applications
}

Dumitru M. Bătineţu-GiURGiU, Dorin MĂRghidAnu and OvidiU T. POP

\section{ABSTRACT.}

In this paper we prove a new generalization of Radon's Inequality and give some applications.

EDITORIAL BOARD OF GAZETA MATEMATICĂ

ACADEMIEI 14

BUCUREŞTI 010014, ROMANIA

E-mail address: dmb_g@yahoo.com

National College "A. I. CuzA"

1 MAI 5

CORABIA 235300, ROMANIA

E-mail address: d.marghidanuegmail.com

National College "Mihai Eminescu"

MIHAI EMINESCU 5

SATU MARE 440014, ROMANIA

E-mail address: ovidiutiberiu@yahoo.com

Received: 22.01.2011; In revised form: 21.09.2011; Accepted: 30.09.2011.

2000 Mathematics Subject Classification. 26D15.

Key words and phrases. Bergström's Inequality, Radon's Inequality. 